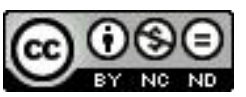

\title{
Atividades Investigativas de Aplicações das Derivadas Utilizando o GeoGebra
}

\section{Investigative Activities Related to Applications of Derivatives Using GeoGebra}

\author{
Daniele Cristina Gonçalves* \\ Frederico da Silva Reis**
}

\begin{abstract}
Resumo
Este artigo apresenta o Produto Educacional gerado a partir de nossa pesquisa de dissertação de mestrado, que aborda as Aplicações das Derivadas, por meio de atividades investigativas utilizando o software GeoGebra. Inicialmente, trazemos uma discussão sobre o ensino de Cálculo, particularmente sobre o ensino de Derivadas e suas aplicações. Em seguida, discutimos a utilização de tecnologias no ensino, aliadas às atividades investigativas, a partir das mudanças que podem ocorrer em sala de aula com a inserção das Tecnologias Informacionais e Comunicacionais na Educação - TICE’s. Após essa breve discussão, apresentamos uma descrição do Produto Educacional constituído e, por fim, algumas considerações sobre sua aplicação e os resultados obtidos, destacando algumas contribuições de atividades investigativas utilizando TICE's para os processos de ensino e aprendizagem de Cálculo I.
\end{abstract}

Palavras-Chave: Ensino de Cálculo e Derivadas. Investigação Matemática. Tecnologias da Informação e Comunicação na Educação.

\footnotetext{
* Mestre em Educação Matemática pela Universidade Federal de Ouro Preto (UFOP). Professora do Sistema de Educação Profissional de Itabira (SEPRO). Endereço para correspondência: Praça da Saúde, 08, Centro, CEP: 35900-495, Itabira, MG, Brasil.E-mail: daniele.goncalves@yahoo.com.br. ${ }^{* * *}$ Doutor em Educação pela Universidade Estadual de Campinas (UNICAMP). Professor Associado do Departamento de Matemática (DEMAT) da Universidade Federal de Ouro Preto (UFOP). Endereço para correspondência: Departamento de Matemática / ICEB / UFOP, Campus Universitário, Morro do Cruzeiro, CEP: 35400-000, Ouro Preto, MG, Brasil. E-mail: fredsilvareis@yahoo.com.br.
} 


\begin{abstract}
This paper presents the educational material that resulted from our master's research on applications of derivatives, through investigative activities using the software GeoGebra. Initially, we present a discussion on the teaching of Calculus, particularly the teaching of derivatives and their applications. We discuss the use of technologies in education, combined with research activities, given the changes that may occur when Information and Communication Technologies in Education - ICTE's are introduced into the classroom. After this brief discussion, we present a description of the educational material developed and, finally, some considerations regarding its implementation and the results obtained, highlighting some contributions using TICE's investigative activities for the teaching and learning of Calculus I.
\end{abstract}

Keywords: Teaching Calculus and Derivatives. Mathematics Research. Information and Communication Technologies in Education.

\title{
1 Sobre o ensino de Cálculo e suas aplicações
}

A partir de uma análise do panorama atual das pesquisas relacionadas ao ensino de Cálculo, podemos perceber que a preocupação por parte dos pesquisadores com o ensino dessa disciplina é crescente no cenário nacional e internacional, onde podemos encontrar um grande número de pesquisas relacionadas ao tema.

As pesquisas em Educação Matemática que tratam do ensino e da aprendizagem do Cálculo se justificam pela importância dessa disciplina em diversos cursos da área de Ciências Exatas. Assim, segundo Igliori (2009, p. 12), "a pesquisa tem papel fundamental no levantamento de causas e na indicação de caminhos a serem trilhados na busca de melhorias".

No que se refere ao ensino de Cálculo, o grande número de pesquisas relacionadas ao tema "se justifica tanto pelo fato de o Cálculo constituir-se um dos grandes responsáveis pelo insucesso dos estudantes quanto por sua condição privilegiada na formação do pensamento avançado em Matemática" (IGLIORI, 2009, p. 13).

Silva e Borges Neto (1994) destacam diversos fatores que interferem no desempenho dos alunos na disciplina de Cálculo. Dentre eles, ressaltam que "o ensino de Cálculo poderia se tornar mais significativo se os professores soubessem em que e como estão sendo aplicados, a posteriori, os conteúdos ensinados" (SILVA; BORGES NETO, 1994, p. 4).

Esses pesquisadores destacam que, muitas vezes, quando questionados 
pelos alunos sobre a importância dos conteúdos estudados em Cálculo, alguns professores não sabem como responder. Muitos têm a convicção de que esta resposta deve ser dada pelos profissionais de disciplinas específicas dos cursos dos alunos, e que seu papel prioritário é trabalhar os conhecimentos matemáticos, desenvolvendo técnicas de resolução de problemas sem ser necessário relacionar o conteúdo com o de outras disciplinas e aplicações que serão ensinadas posteriormente.

Barbosa (2004, p. 11) critica essa postura dos professores: "O Cálculo pelo cálculo, sem aplicação e contextualização, fica centrado em uma pedagogia rotineira, tradicional, em que muitos docentes estão acostumados".

As aplicações dos conteúdos do Cálculo podem ser úteis, também, como formas de motivação do estudante. Pela experiência de Silva e Borges Neto (1994), quando os alunos conseguem relacionar os conteúdos com situações reais que possam ser vivenciadas em sua vida profissional, o nível de interesse é maior, proporcionando melhor apreensão dos conhecimentos trabalhados e, com isso, as habilidades são desenvolvidas mais rapidamente.

A pesquisa de Catapani (2001) mostra outra ótica desse fato. Em entrevistas realizadas pela pesquisadora com professores de disciplinas específicas do curso de Geologia, um dos pontos de destaque está na falta de articulação e integração entre os departamentos dentro da universidade. A hipótese apresentada por um entrevistado é de que a interação entre os professores de Cálculo e os professores de matérias específicas de Geologia pode contribuir para uma proposta que enfatize atividades relacionadas a aplicações dos conteúdos do Cálculo no curso em que o aluno está inserido.

Outros fatores também são destacados pelos professores entrevistados, como o descompasso entre a disciplina de Cálculo e as disciplinas específicas do curso em questão:

De acordo com esses professores, embora o aluno de curso de Geologia, na maioria das vezes, não tenha consciência da importância e da necessidade da disciplina Cálculo Diferencial e Integral, ela se faz muito importante como linguagem e como instrumento na resolução dos problemas da área. Técnicas de derivada e integral, segundo afirmam, são de grande utilidade nas várias disciplinas, e estão presentes no desenvolvimento e no entendimento dos processos, isso tudo, além de considerar o processo de quantificação, cada vez mais necessário na área em questão (CATAPANI, 2001, p. 53). 
Dessa forma, cabe ao professor de Cálculo, nos anos iniciais do curso, apresentar ao estudante a importância da disciplina no curso que este está inserido. Concebemos que uma possível forma de mostrar essa importância é desenvolvendo atividades em sala de aula em que sejam trabalhadas as aplicações.

\section{Sobre o ensino de derivadas no Cálculo}

O conceito de derivada é considerado um dos conceitos fundamentais do Cálculo. Por isso, seu estudo está presente no currículo de diversos cursos superiores, dentro de disciplinas relacionadas ao Cálculo, por possuir aplicações em várias áreas do conhecimento. Segundo Zuin (2001), as derivadas estão presentes em diversas situações cotidianas relacionadas ao movimento e à variação.

O próprio desenvolvimento do Cálculo e de seus conceitos fundamentais tem sua origem a partir de situações reais. Seu surgimento ocorreu a partir da busca de soluções para problemas como:

Calcular a distância percorrida por um corpo em movimento, sua velocidade e aceleração; comprimentos de curvas; áreas; volumes; analisar os valores de máximo e mínimo de uma função; relacionar declividade de uma curva e taxa de variação, são alguns dos problemas, entre muitos outros, que levaram ao desenvolvimento do Cálculo (ZUIN, 2001, p. 14).

No entanto, a derivada tem sido um dos tópicos do Cálculo Diferencial e Integral em que os estudantes apresentam muitas dificuldades de aprendizagem. As pesquisas relacionadas ao ensino de derivadas sinalizam que há problemas nesse processo e apontam caminhos a serem seguidos para superar essas dificuldades intrínsecas ao processo (CATAPANI, 2001; BARBOSA, 2004).

Baseada em sua experiência, Villarreal (1999, p. 7) relata que "o conceito de derivada mostra-se como uma noção que apresenta dificuldades frequentes e persistentes para os estudantes e sua compreensão é de fundamental importância nos cursos de Cálculo".

A derivada é um conceito que pode ser explorado a partir de diversos focos: derivada como um limite, como inclinação da reta tangente a uma curva em um ponto dado, além de situações que envolvem taxa de variação, máximos e mínimos. Uma das possíveis causas para as dificuldades dos alunos na aprendizagem do conceito de derivada pode estar relacionada a dificuldades na 
aprendizagem de limites, que acarretam, como consequência, dificuldades em derivadas, decorrentes do fato de a derivada ser um limite.

D’Avoglio (2002) identificou essa dificuldade na compreensão do conceito de derivada de uma função em um ponto quando definida de modo formal, a partir do conceito de limite. Objetivando verificar qual era o conhecimento sobre o conceito de derivada de alunos que já haviam estudado o assunto, aplicou um teste de sondagem e identificou que alguns alunos confundem:
a) derivada com reta tangente,
b) derivada num ponto com a função derivada,
c) derivada com regra para se achar derivada,
d) reta tangente com coeficiente angular da reta tangente e também, que muitos apresentam dificuldade de expressão (D’AVOGLIO, 2002, p. 27, grifos do autor).

Os resultados da pesquisa de D'Avoglio (2002) apontaram evidências de que a introdução do conceito de derivada de uma função em um ponto, partindo do conceito de velocidade, por ser algo conhecido do aluno, contribuiu positivamente para a aprendizagem destes. Ao ser possível associar um conhecimento anterior ao novo conhecimento, o novo conceito passou a ter algum significado, despertando, assim, o interesse dos alunos.

Isso é relevante, pois o estudante do curso universitário está em busca de uma formação sólida que o capacite para o mercado de trabalho. Dessa forma, os alunos procuram um conhecimento que seja útil em sua futura vida profissional. Considerando esse perfil, é importante que o estudante conheça onde se aplicam os conceitos trabalhados pelo professor de Cálculo, para que os conteúdos estudados passem a ter algum significado prático.

Nossa concepção é de que os estudantes precisam conhecer os instrumentos do Cálculo, mas também devem ser explorados os conceitos e as aplicações. Quando utilizamos o termo aplicações, referimo-nos a situaçõesproblema relacionadas a qualquer área do conhecimento que podem ser resolvidas utilizando ferramentas e conceitos matemáticos. Idealizamos, dessa forma, uma prática em sala de aula contextualizada, como defende Barbosa (2004):

Essa prática contextualizada exige do aluno uma associação de vários conteúdos estudados em outras disciplinas, bem como práticas vivenciadas. Por outro lado, o professor, enquanto articulador, mediador e aprendiz, amplia seus conhecimentos, gerando assim uma prática cotidiana mais significativa para o ensino do Cálculo Diferencial e Integral (BARBOSA, 2004, p. 42). 
Em relação ao ensino de derivadas, podemos perceber que o foco dos alunos, muitas vezes, fica restrito à aplicação de regras de derivação. Desse modo, quando é necessária a utilização de algum conceito para o desenvolvimento de alguma atividade, os alunos geralmente apresentam dificuldades em sua realização. Uma possível causa para esse fato é que os alunos não estão acostumados a resolver situações em que seja necessária a utilização / aplicação dos conceitos.

A pesquisa de Barbosa (2004) identificou que a maior parte dos alunos por ele investigados dedica poucas horas extraclasse para os estudos, e, quando estudam, muitas vezes o foco é dado à resolução de exercícios, geralmente resolvidos de forma mecânica, somente aplicando regras sem significados.

Como professores de Cálculo, sabemos que o aluno, sozinho, muitas vezes não é capaz de estabelecer relação entre as disciplinas matemáticas e sua aplicação prática em seu curso. Barbosa (2004) afirma:

A contextualização do saber é uma ferramenta indispensável para a questão da transposição didática, pois implica recorrer a contextos que tenham significado para o aluno, envolvendo-o não só intelectualmente, mas também afetivamente, sendo assim uma estratégia fundamental para a construção de significados. Sabemos que a falta de sentido da aprendizagem de Cálculo Diferencial e Integral origina-se, em parte, das dificuldades decorrentes dessa transposição. O aluno só compreende os vínculos do conteúdo estudado quando fica compreensível para ele essa passagem. Por isso, contextualizar no ensino de Cálculo vincularia os conhecimentos aos lugares onde foram criados e onde são aplicados, isto é, incorporar vivências concretas ao que se vai aprender e incorporando o aprendizado a novas vivências (BARBOSA, 2004, p. 41).

Essa é uma dificuldade também enfrentada pelos professores de Matemática que, geralmente, têm uma formação matemática formal e rigorosa, eles mesmos desconhecendo as aplicações. Embora a formação do docente em Matemática, muitas vezes, não o desperte em relação à aplicação dos conteúdos, cabe ao professor da disciplina de Cálculo buscar esse conhecimento para ser explorado em sala de aula. As aplicações são, assim, uma forma de estimular e motivar os estudantes. 


\section{Sobre as aplicações das derivadas}

Notamos que há uma carência de pesquisas que tratem das aplicações das derivadas. Esse fato foi também percebido por Ramos (2009), que realizou uma pesquisa com alunos do curso de Licenciatura em Matemática relacionada às aplicações das derivadas, sendo que estes já haviam cursado a disciplina de Cálculo I, quando esse tópico havia sido estudado.

Essa pesquisa foi realizada com o intuito de investigar o conhecimento dos alunos sobre as aplicações das derivadas e classificar suas dificuldades. Para isso, o pesquisador elaborou uma sequência de quatro atividades, estruturada a partir da teoria dos Registros de Representação Semiótica de Raymond Duval.

De acordo com esse referencial, é importante que o professor proponha atividades em que o aluno utilize as diversas formas de representação dos objetos matemáticos e saiba fazer a conversão entre uma forma de registro e outra. Desse modo, se o aluno possui o domínio das diversas formas de representação de um objeto matemático, ele pode optar pelo mais adequado na resolução de um problema proposto.

Nesse sentido, concordamos com o pesquisador, visto que consideramos a importância de se estudar o conceito de derivada não somente de forma algébrica, mas, também, geométrica. Em nossa prática, percebemos que, muitas vezes, o que predomina para o aluno é a derivada como resultado de um processo operatório. Concebemos que quando os cálculos de derivadas são realizados algebricamente, mas associados à sua interpretação geométrica, é possível que o aluno estabeleça uma relação entre as operações realizadas e o conceito.

As atividades propostas por Ramos (2009) exploram os diversos registros de representação da derivada de uma função real, como o registro algébrico, gráfico e geométrico, além de sugerir que o estudante realize a conversão entre uma forma de representação e outra. Em alguns tópicos das atividades, é necessária a utilização do conceito de derivada e da relação entre o gráfico de uma função e o de suas derivadas, em relação a crescimento, decrescimento, máximo e mínimo local e pontos de inflexão.

Ramos (2009, p. 42) destaca o foco fundamental de sua pesquisa, que "é analisar se, de fato, após um curso de Cálculo, as competências elementares sobre as aplicações da derivada fazem parte das habilidades adquiridas pelos estudantes". A partir da análise dos dados, observou que os alunos têm facilidade nas tarefas em que são necessárias manipulações algébricas.

Quanto às situações que envolvem registros gráficos, foram percebidas 
dificuldades em identificar os dados contidos nessa forma de representação e estabelecer relações com o registro algébrico, além de deficiências conceituais relacionadas ao comportamento do gráfico de uma função e de suas derivadas. O pesquisador identificou, também, dificuldades em situações onde é necessária a utilização do conceito de derivada e, igualmente, que muitos alunos não conseguem traçar estratégias para a resolução de situações que envolvem aplicações.

O pesquisador conclui que as dificuldades apresentadas pelos estudantes são tanto de caráter conceitual quanto manipulativas:

[...] pois eles efetuam tratamentos e chegam a resultados, mas, ora não sabem identificar as relações deles com o comportamento gráfico de uma função, ora não conseguem aplicar o conceito de derivada para efetuar os tratamentos (RAMOS, 2009, p. 81).

Quanto às situações que envolvem aplicações das derivadas, as dificuldades "se alternam em conceituais e manipulativas, conforme o tipo de registro de representação utilizado na situação de aplicação proposta" (RAMOS, 2009, p. 82). O pesquisador ressalta a necessidade de os professores trabalharem mais as aplicações da derivada com seus alunos, pois ele tem a convicção de que "quando o aluno aplica o novo conhecimento, compreende melhor o conceito".

\section{As TICE's e a investigação matemática}

A utilização de ferramentas computacionais em sala de aula possui grande potencial pedagógico, que pode auxiliar o professor na tarefa de ensinar, possibilitando ao educando um conhecimento dinâmico. Segundo Gravina e Santarosa (1998), um ambiente educacional informatizado possibilita ao aluno a construção do seu conhecimento, pois com o auxílio de um recurso computacional o estudante pode modelar problemas e fazer simulações, além de visualizar uma situação, o que, muitas vezes, não seria possível sem essa ferramenta.

A utilização de software permite que os conceitos matemáticos sejam explorados por meio de construções não estáticas, que podem ser manipuladas e proporcionar uma percepção diferente da Matemática.

Ambientes informatizados são propícios para a realização de uma atividade investigativa, pois o aluno pode ser levado a explorar situações, a formar o próprio pensamento e a investigar. Para Ponte, Brocardo e Oliveira (2006, p. 
13) "investigar é descobrir relações entre objetos matemáticos conhecidos ou desconhecidos, procurando identificar as respectivas propriedades". Tais ambientes permitem ao aluno analisar uma situação e observar regularidades, estabelecer hipóteses e testá-las na busca de uma solução para o problema proposto.

Ponte, Brocardo e Oliveira (2006, p. 25) afirmam, ainda, que "as investigações matemáticas são um tipo de atividade que todos os alunos devem experimentar". Assim, ao educador, enquanto mediador da aprendizagem, cabe explorar, junto com os estudantes, o conhecimento matemático e os conceitos envolvidos. A observação e a percepção podem ser estimuladas para desenvolver nos alunos a capacidade de criticar e questionar a Matemática como um conhecimento em construção. É importante incentivar, também, a justificação, para promover no educando a capacidade de argumentação das suas ideias.

Segundo Borba e Penteado (2001), a utilização de software possibilita a experimentação com conteúdos matemáticos, além de estimular a percepção visual do aluno. Partindo de uma imagem, pode-se explorar o conceito matemático envolvido em uma situação problema.

A visualização é uma questão muito explorada por pesquisadores das tecnologias na Educação Matemática. Costa (2002, p. 262), por exemplo, apresenta definições de diversos autores sobre "visualização", também identificada como "pensamento visual", destacando que há um consenso em que a "visualização se foca na percepção e na manipulação de imagens visuais".

Para Couy (2008), quando imagens visuais são manipuladas, a Matemática adquire um caráter exploratório, sendo possível analisar, interpretar, descobrir variantes e compreender o conteúdo matemático, suas características e propriedades, estimulando a descoberta. A pesquisadora defende "uma abordagem visual dos conceitos, numa interlocução com as demais formas de representação em Matemática" (COUY, 2008, p. 47), e destaca que a visualização é, atualmente, considerada um processo importante na aprendizagem matemática, mas ainda pouco utilizada em sala de aula.

Além disso, segundo Couy (2008), a utilização de software no ensino de Matemática possibilita uma relação de troca entre o aluno e a Matemática. Ao se deparar com uma situação-problema, se o aluno dispõe de alguma ferramenta computacional, seu trabalho pode ficar mais agradável e interativo:

Ferramentas tecnológicas, se utilizadas de forma adequada, podem potencializar o uso dos recursos gráficos no ensino de Cálculo, estimulando a observação, a busca de 
regularidades e padrões e possibilitando, através da comparação com as outras formas de se representar uma função, o entendimento das ligações entre elas. O trabalho desenvolvido com a utilização desses recursos também pode contribuir para que os alunos apurem a percepção e, por consequência, desenvolvam habilidades que facilitem a construção gráfica por meio dos instrumentos tradicionais: lápis, papel e régua (COUY, 2008, p. 47).

Por outro lado, a presença das ferramentas computacionais nas aulas de Matemática não implica no abandono de outros instrumentos educacionais. A informática é um recurso auxiliar que possibilita o alcance dos resultados na aprendizagem, por meio do seu uso adequado e conciliação das diversas formas de se ensinar e aprender, com o professor e o aluno desempenhando seus papéis. Tudo isso considerando que, sozinha, a ferramenta computacional não produz conhecimento (GRAVINA; SANTAROSA, 1998).

De fato, segundo Gravina e Santarosa (1998), é necessário ter cuidados com a utilização da informática em sala de aula, para que as atividades não sejam limitadas à repetição de exercícios, destacam que o software não pode dificultar a realização de uma tarefa devido ao não conhecimento de suas ferramentas.

As TICE's têm se constituído um recurso didático muito importante no ensino de Cálculo e sua utilização tem sido muito recomendada por pesquisadores em Educação Matemática (MARIN, 2009), pelo fato de permitir ao professor explorar diversos conceitos matemáticos e representações de maneira rápida e eficaz.

Segundo Reis (2001, 2009), muitos conceitos do Cálculo são trabalhados na sala de aula de forma clássica, por meio de definições, teoremas, demonstrações e propriedades, seguindo-se listas de exercícios para os alunos resolverem. Dessa forma, muitas vezes, os alunos se preocupam somente em realizar operações algébricas e em memorizar fórmulas, não dando muita atenção aos conceitos.

Para Villarreal (1999), alguns conceitos do Cálculo carregam em si uma dinamicidade que, muitas vezes, não é possível de ser observada utilizando-se apenas quadro e giz e/ou pincel. Utilizando-se de uma ferramenta computacional, os conceitos da disciplina podem ser explorados de forma a facilitar a compreensão dos mesmos. De acordo com Villarreal (1999): 
A presença do computador oferece a possibilidade de observar processos de construção de conhecimento matemático que não apareceriam em outros ambientes e que vão além do simples uso do computador para resolver um determinado problema matemático (VILLARREAL, 1999, p. 28).

Segundo Marin (2009), a utilização de recursos computacionais na sala de aula pode contribuir não só para a abordagem conceitual na disciplina de Cálculo, mas, também, com outros aspectos, como um maior tempo disponível para exploração e investigação, uma vez que a ferramenta computacional dispensa a realização de cálculos trabalhosos e permite ao aluno mais autonomia em relação ao seu aprendizado, facilitando o entendimento do conteúdo dessa disciplina, considerada difícil por muitos pesquisadores. Além disso, o uso de tecnologias, principalmente no ensino de Cálculo, pode expandir possibilidades de trabalho com diferentes abordagens e representações algébricas e geométricas, de forma rápida e articulada (MARIN, 2009).

$\mathrm{O}$ pesquisador ressalta, igualmente, que a tecnologia "permite realizar atividades que seriam impossíveis de serem feitas somente com o uso de lápis e de papel, proporcionando a organização de situações pedagógicas com maior potencial para aprendizagem" (MARIN, 2009, p. 136).

Outro aspecto interessante a se destacar é que alguns livros didáticos de Cálculo sugerem a utilização da informática como recurso auxiliar, além do livro didático. Stewart (2011), no prefácio de seu livro afirma que:

A disponibilidade de tecnologia não diminui - pelo contrário, aumenta - a importância de se entender com clareza os conceitos por trás das imagens na tela. Quando utilizados apropriadamente, computadores e calculadoras gráficas são ferramentas úteis na descoberta e compreensão de tais conceitos (STEWART, 2011, p. vii).

Além disso, ainda segundo Marin (2009), com o uso da informática os alunos se preocupam menos com as operações e a parte técnica, possibilitando ao professor uma exploração diferenciada do conteúdo, privilegiando a compreensão e interpretação. Assim, os alunos "são levados de uma maneira rápida a tentar coisas diferentes, a buscar novas descobertas, a observar propriedades, a testar parâmetros, a investigar de maneira diferente da qual estão habituados" (MARIN, 2009, p. 138). 


\section{Descrição do produto educacional}

O Produto Educacional que apresentamos contém uma sugestão de atividades investigativas para o ensino de Aplicações das Derivadas com a utilização de um software gráfico. Ele é o resultado gerado a partir de nossa Dissertação do Mestrado Profissional em Educação Matemática, do programa de pós-graduação da Universidade Federal de Ouro Preto (UFOP), intitulada Aplicações das Derivadas no Cálculo I: Atividades investigativas utilizando $o$ GeoGebra, sob a orientação do Prof. Dr. Frederico da Silva Reis.

Nosso objetivo é apresentar um material que complementa e revitaliza alguns exercícios e atividades clássicos de aplicações de derivadas que são, tradicionalmente, encontrados nos livros-textos, muito utilizados nas referências das disciplinas de Cálculo I nas universidades brasileiras, tais como Stewart (2011) e Thomas (2011). Para elaborarmos as atividades, inicialmente, analisamos a natureza dos exercícios propostos por cada autor, relacionados às aplicações das derivadas. Em seguida, selecionamos quatro exercícios que julgamos ter potencial para a investigação. Daí, adaptamos os textos introdutórios com o objetivo de contextualizar cada exercício e inserimos algumas orientações para a utilização do software GeoGebra, sempre com a finalidade de tornar os exercícios verdadeiras atividades investigativas, dentro da perspectiva adotada em nossa pesquisa.

Buscando identificar contribuições do desenvolvimento de atividades investigativas com auxílio de TICE's para os processos de ensino e aprendizagem de Cálculo I, no que se refere às aplicações das derivadas, elaboramos as atividades na perspectiva de uma investigação que privilegie as "relações entre objetos matemáticos, procurando identificar propriedades" (PONTE; BROCARDO; OLIVEIRA, 2006, p. 13), tendo como características "a descoberta, a exploração, a pesquisa e a autonomia" (PORFÍRIO; OLIVEIRA, 1999, p. 111) e, principalmente, enfatizando "processos matemáticos tais como procurar regularidades, formular, testar, justificar e provar conjecturas, refletir e generalizar" (OLIVEIRA; SEGURADO; PONTE, 1999, p. 191).

As atividades apresentadas em nosso Produto Educacional foram aplicadas a alunos de uma turma da disciplina Cálculo Diferencial e Integral I do curso de Licenciatura em Matemática de uma universidade pública. São elas: Atividade 1- Construindo um tanque cilíndrico; Atividade 2- Delimitando uma reserva; Atividade 3- Projetando um tanque retangular; Atividade 4Projetando uma caixa. 
Foi feita a escolha pelo software GeoGebra por se tratar de um software gratuito, com uma interface amigável, disponibilizando simultaneamente as representações algébrica e geométrica, além de possuir recursos de dinamicidade e movimentação.

Esperamos que esse material possa contribuir, de forma significativa, para os processos de ensino e aprendizagem de Cálculo I, bem como propiciar reflexões a respeito de nossa postura como professores/educadores matemáticos.

Os Produtos Educacionais gerados a partir das pesquisas realizadas no programa de pós-graduação da Universidade Federal de Ouro Preto são disponibilizados, de forma gratuita, na página do programa, disponíveis em <http:/ /www.ppgedmat.ufop.br>. Acesso em: 08 ago. 2013. Além disso, o Instituto de Ciências Exatas e Biológicas (ICEB) da Universidade Federal de Ouro Preto promove, anualmente, o Encontro de Ensino e Pesquisa em Educação Matemática, com o objetivo de divulgar os Produtos gerados a partir das pesquisas de dissertação de mestrado, na forma de minicursos, ministrados pelos autores. Os Produtos Educacionais gerados são também publicados na forma de livreto pela Editora UFOP.

\section{Considerações sobre a aplicação do Produto Educacional e seus resultados}

Com base em nossa pesquisa realizada (GONÇALVES, 2012), pudemos inferir algumas categorias de contribuições de atividades investigativas utilizando TICE's para os processos de ensino e aprendizagem de Cálculo I:

1) A contribuição para a ressignificação dos conhecimentos dos alunos em relação às aplicações das derivadas: nossa pesquisa apontou que a realização das atividades investigativas contribuiu para uma ressignificação dos conhecimentos dos alunos, inicialmente construídos em sala de aula, a partir da oportunidade que eles tiveram de repensar/refletir sobre os conceitos envolvidos nas diversas aplicações das derivadas abordadas nas atividades. Reafirmamos, então, nossa crença de que o desenvolvimento de atividades investigativas utilizando TICE's pode contribuir para a construção e ressignificação de conceitos nucleares do Cálculo Diferencial e Integral.

2) A contribuição para a criação de um ambiente de aprendizagem diferenciado e complementar à sala de aula: nossa pesquisa apontou que a realização das atividades investigativas contribuiu para a criação de um ambiente de discussão, conjecturação e colaboração que nem sempre é possível de se ter 
na sala de aula tradicional, na qual o processo de aprendizagem é, quase sempre, totalmente guiado pelo professor. Reafirmamos, então, nossa crença de que o desenvolvimento de atividades investigativas utilizando TICE's pode contribuir para a criação de um ambiente informatizado de aprendizagem que complementa a sala de aula.

3) A contribuição para a formação de um "novo" professor de Matemática dos Ensinos Fundamental e Médio e também do Ensino Superior: nossa pesquisa apontou que a realização das atividades investigativas contribuiu para formação inicial dos alunos participantes, futuros professores de Matemática dos Ensinos Fundamental e Médio, na medida em que eles tiveram a oportunidade de refletir sobre a importância da realização de atividades com software para o futuro professor de Matemática. Reafirmamos, então, nossa crença de que o desenvolvimento de atividades investigativas utilizando TICE's pode contribuir para os processos de ensino e aprendizagem de Cálculo I que é fundamental na formação do professor de Matemática.

\section{Referências}

BARBOSA, M. A. O insucesso no ensino e aprendizagem na disciplina de Cálculo Diferencial e Integral. 2004. 101f. Dissertação (Mestrado em Educação) - Pontifícia Universidade Católica do Paraná, Curitiba, 2004.

BORBA, M. C.; PENTEADO, M. G. Informática e Educação Matemática. Belo Horizonte: Autêntica, 2001.

CATAPANI, E. C. Cálculo em serviço: um estudo exploratório. Bolema, Rio Claro, v. 14, n. 16, p. 48-62, 2001.

COSTA, M. C. M. Processos mentais associados ao pensamento matemático avançado: Visualização. In: PONTE, J. P. (Org.). Atividades de investigação na aprendizagem da Matemática e na formação de professores. Coimbra: Escola Superior de Educação de Coimbra, 2002. p. 257-273.

COUY, L. Pensamento visual no estudo da variação de funções. 2008. 160f. Dissertação (Mestrado em Ensino de Ciências e Matemática) - Pontifícia Universidade Católica de Minas Gerais (PUC Minas), Belo Horizonte, 2008.

D'AVOGLIO, A. R. Derivada de uma função num ponto: uma forma significativa de introduzir o conceito. 2002. 91f. Dissertação (Mestrado em Educação Matemática) Pontifícia Universidade Católica de São Paulo, São Paulo, 2002. 
GONÇALVES, D. C. Aplicações das Derivadas no Cálculo I: atividades investigativas utilizando o GeoGebra. 2012. 110f. Dissertação (Mestrado em Educação Matemática) Universidade Federal de Ouro Preto, Ouro Preto, 2012.

GRAVINA, M. A.; SANTAROSA, L. M. A aprendizagem da Matemática em ambientes informatizados. In: CONGRESSO IBERO-AMERICANO DE INFORMÁTICA NA EDUCAÇÃO, 4., 1998, Brasília. Anais... Brasília: RIBIE, 1998. Disponível em: <lsm.dei.uc.pt/ribie/docfiles/txt200342413933117.PDF>. Acesso em: 03 abr. 2010.

IGLIORI, S. B. C. Considerações sobre o ensino de Cálculo e um estudo sobre números reais. In: FROTA, M. C. R.; NASSER, L. (Org.). Educação Matemática no Ensino Superior: pesquisas e debates. Recife: SBEM, 2009. p.11-26.

MARIN, D. Professores de Matemática que usam a tecnologia de informação e comunicação no ensino superior. 2009. 164f. Dissertação (Mestrado em Educação Matemática) - Universidade Estadual Paulista, Rio Claro, 2009.

OLIVEIRA, H. M.; SEGURADO, M. I.; PONTE, J. P. Tarefas de investigação em Matemática: histórias da sala de aula. In: ABRANTES, P. et al. (Org.). Investigações matemáticas na aula e no currículo. Lisboa: Projeto MPT e APM, 1999. p.189-206.

PONTE, J. P.; BROCARDO, J.; OLIVEIRA, H. Investigações matemáticas na sala de aula. Belo Horizonte: Autêntica, 2006.

PORFÍRIO, J.; OLIVEIRA, H. Uma reflexão em torno das tarefas de investigação. In: ABRANTES, P. et al. (Org.). Investigações matemáticas na aula e no currículo. Lisboa: Projeto MPT e APM, 1999. p.111-118.

RAMOS, V. V. Dificuldades e concepções de alunos de um curso de licenciatura em Matemática sobre derivada e suas aplicações. 2009. 84f. Dissertação (Mestrado em Educação Matemática) - Pontifícia Universidade Católica de São Paulo, São Paulo, 2009.

REIS, F. S. A Tensão entre Rigor e Intuição no Ensino de Cálculo e Análise: A visão de professores-pesquisadores e autores de livros didáticos. 2001. 302f. Tese (Doutorado em Educação) - Universidade Estadual de Campinas, Campinas, 2001.

REIS, F. S. Rigor e intuição no ensino de Cálculo e Análise. In: FROTA, M. C. R.; NASSER, L. (Org.). Educação Matemática no Ensino Superior: pesquisas e debates. Recife: SBEM, 2009. p. 81-97. 
SILVA, J. F.; BORGES NETO, H. Questões Básicas do Ensino do Cálculo. Artigo Científico. Laboratório de Pesquisa Multimeios da Faculdade de Educação da Universidade Federal do Ceará, 1994. Disponível em: <http://www.multimeios.ufc.br/ arquivos/pc/artigos/artigo-questoes-basicas-do-ensino-de-calculo.pdf $>$. Acesso em: 03 mar. 2011.

STEWART, J. Cálculo. Volume I. São Paulo: Thompson Learning, 2011.

THOMAS, G. B. Cálculo I. São Paulo: Addison Wesley, 2011.

VILLARREAL, M. E. O pensamento matemático de estudantes universitários de Cálculo e tecnologias informáticas. 1998. 387f. Tese (Doutorado em Educação Matemática) - Universidade Estadual Paulista, Rio Claro, 1999.

ZUIN, E. S. L. Cálculo: uma abordagem histórica. In: LAUDARES, J. B.; LACHINI, J. (Org.). Educação Matemática: a prática educativa sob o olhar de professores de Cálculo. Belo Horizonte: FUMARC, 2001. p. 13-36.

Submetido em Agosto de 2012. Aprovado em Novembro de 2012. 